Discharge kinetics and emission characteristics of a large-area-cold cathode flash X-ray tube: parametric study and numerical modelling

This content has been downloaded from IOPscience. Please scroll down to see the full text.

1990 J. Phys. D: Appl. Phys. 2318

(http://iopscience.iop.org/0022-3727/23/1/004)

View the table of contents for this issue, or go to the journal homepage for more

Download details:

IP Address: 134.76.223.157

This content was downloaded on 04/01/2017 at 13:39

Please note that terms and conditions apply.

You may also be interested in:

Simple flash X-ray tubes with a new type of cathode

F Adolfs, F Muller and E Schulte

An intense-current electron beam source with low-level plasma formation

Limin Li, Lie Liu, Jianchun Wen et al.

First results of KALI-30 GW with 1 MV flash X-rays generation and characterization by Imaging plate

A Sharma, A M Shaikh, K Senthil et al.

The laser triggering of high-voltage switches

A H Guenther and J R Bettis

A High Intensity Flash X-ray Tube

A Mattsson

Excitation of a rare-gas halide laser by electron beam pulses of microsecondduration

Yu I Bychkov, N G Ivanov, I N Konovalov et al.

$\underline{X-r a y ~ s p o t s ~ e m i t t e d ~ i n ~ a ~ h o l l o w ~ c a t h o d e ~ n s-d i s c h a r g e ~}$

M Skowronek, A Ikhlef, G Louvet et al.

KrF-laser-triggered switching of a multi-line pulsed power system

C B Edwards, F O'Neill and M J Shaw 


\title{
Discharge kinetics and emission characteristics of a large-area cold cathode flash $x$-ray tube: parametric study and numerical modelling
}

\author{
M Steyer† \\ Max-Planck-Institut für Biophysikalische Chemie, Abt. Laserphysik, Postfach 2841 , \\ D-3400 Göttingen, FRG
}

Received 14 June 1989, in final form 31 July 1989

\begin{abstract}
A pulsed $x$-ray tube is described which consists of a large-area $\left(80-400 \mathrm{~cm}^{2}\right)$ cold carbon felt cathode and a tantalum foil anode arranged in transmission geometry. It is driven by a Marx generator with an output voltage between 60 and $140 \mathrm{kV}$. The dose and the dose rate of the $\mathrm{x}$-ray flash together with the diode current are studied as a function of anode-cathode distance, the cathode area and the charging voltage. The tube characteristics are numerically well described by a simple model which considers cathode plasma migration and a space-charge limited current in the remaining vacuum gap.
\end{abstract}

\section{Introduction}

X-ray flash sources are widely used for the observation of fast phenomena. Depending on the application, high repetition rate, high dose, short pulse duration or high intensity are demanded [1,2]. More recently, x-ray flash tubes have also been used for the preionisation of high-pressure gas discharge lasers [3-7]. If a high $\mathrm{x}$ ray intensity is required, cold cathodes are often employed, because they are capable of a space-charge limited current, which can be considerably higher than that of thermoionic emitters [8].

Compared with commonly used metal blades, cathodes made of carbon fibres or carbon felt have been found to be advantageous in vacuum discharges with regard to their $\mathrm{x}$-ray emission homogeneity and lifetime [9]. Due to the high number of potential fieldemitting whiskers, statistical fluctuations and the evaporation of single whiskers are of minor importance. Additionally, carbon felt cathodes are scalable to large areas as required in electron beam pumped or $\mathrm{x}$-ray preionised laser technology, where they have become a widely used technique $[5,7]$.

Despite their widespread use, there have been only a few attempts to explain the operational characteristics of cold cathode $\mathrm{x}$-ray tubes in the pre-breakdown phase by analytical or numerical models [7, 10-12].

In this paper we present a detailed study of an $x-$ ray flash tube consisting of a Marx generator and a

$\uparrow$ Present address: Central Laser Facility, Rutherford Appleton Laboratory, Chilton, Didcot, Oxon OX11 0QX, UK. vacuum diode employing a carbon felt cathode with a large emission area $\left(80-400 \mathrm{~cm}^{2}\right)$. The dependence of tube current and $\mathrm{x}$-ray output on cathode area, electrode separation and generator design is investigated. A non-linear, second-order differential equation describes the circuit including the Marx generator, the cables and the vacuum diode. Under the assumption of a constantly moving cathode plasma with increasing effective emission area, the tube current and $x$-ray output during plasma migration are calculated in good agreement with experimental data.

\section{Experiment}

A schematic diagram of the flash $x$-ray system consisting of the high-voltage generator and the vacuum diode is shown in figure 1 . The diode chamber is made of $6 \mathrm{~mm}$ thick stainless steel with a $10 \times 40 \mathrm{~cm}^{2}$ large $\mathrm{Al}$ output window of $3 \mathrm{~mm}$ thickness at the bottom and lead glass windows for discharge observation at the side walls. The cathode is made of $3 \mathrm{~mm}$ thick and $40 \mathrm{~cm}$ long carbon felt (1) while the anode consists of a $10 \mu \mathrm{m}$ thick tantalum foil (2). Both electrodes are fixed to their supports by conductive glue. Optionally, a peaking capacitor $C_{\mathrm{p}}$ is formed by a steel plate in contact with the high-voltage feedthrough and the tube housing with deionised water as the dielectric material (3). Two cathode sizes of $2 \mathrm{~cm}$ and $10 \mathrm{~cm}$ width have been tested. The chamber is evacuated to $\approx 2 \times 10^{-5}$ mbar. The tube is powered by a $n$-stage $(n=3-4)$ Marx 


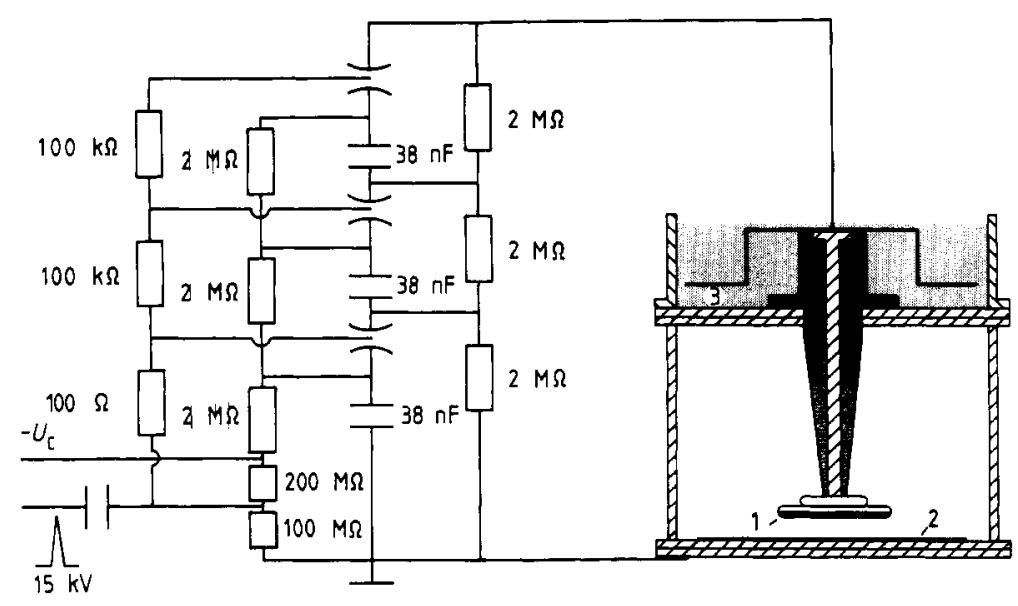

Figure 1. Schematic diagram of the $x$-ray flash system (see text for details).

generator, each stage consisting of a $38 \mathrm{nF}$ capacitor and an electrically triggered spark gap (Maxwell). Each stage can be charged up to $U_{\mathrm{C}}=35 \mathrm{kV}$. The current through the diode was measured with a current transformer (Pearson Model 110). A PIN diode (UDT pin 10) protected against visible light with a thin aluminium foil was used to trace the $\mathrm{x}$-ray pulse $[6,13]$. Since the active layer of this detector is very thin $(\approx 250 \mu \mathrm{m})$ it is reasonable to assume that it measures the $\mathrm{x}$-ray dose rate.

The $\mathrm{x}$-ray dose was determined with a pocket dosemeter FH 39 R5 (FAG) at a distance of $3 \mathrm{~cm}$ from the output window. Since the $\mathrm{x}$-ray beam is highly divergent, both PIN diode and dosemeter were enclosed in a small lead box so that they only collect the $\mathrm{x}$-rays emitted in the forward direction.

The electrical signals were recorded with a 7834 storage oscilloscope or with a 7250 transient digitiser (Tektronix). The tube voltage at the high-voltage feedthrough was monitored with a resistive voltage divider (made available by Dr A Endo, Tokyo) which has a temporal resolution of about 50 ns. Little effort was made to minimise the inductance of the electrical circuit, and depending on the actual geometrical current loop size, it amounts to a few microhenry.

\section{Discharge mechanism}

Vacuum discharges between cold cathodes have been extensively studied $[11,12,14-17]$ and the principles of operation are well understood. However, due to the high number of parameters which are difficult to quantify (e.g., surface roughness of the electrodes), there is still a high degree of empiricism in the description of vacuum discharges $[2,15]$. It is commonly agreed that the phenomena occurring during arc formation are exceedingly complex [18]. Let us briefly review the most important processes for the present application.

Depending on the shape and material of the cold cathode, the geometry and the background gas pressure, electrons are extracted either directly from the cathode (field-emission tube) or from a plasma formed by evaporation of cathode material (vacuum discharge tube) [2]. A very low background pressure $\left(\approx 10^{-10}\right.$ mbar $)$ is needed to achieve a pure fieldemission current while the requirement for effective $\mathrm{x}$ ray production via plasma emission is quite moderate $\left(\approx 10^{-5}\right.$ mbar) $[2,8]$. At large inter-electrode separation (several centimetres) a pure field-emission current is not likely to occur [2]. The evaporation in vacuum discharge tubes occurs due to Joule heating of protrusions by high local field-emission currents [14-16]. The vapour thus produced is partially ionised by electrons, and positive ion bombardment of the cathode enhances the electron emission [18]. A highly conductive plasma is formed, which works as an effective cathode with a very small work function [16]. Additionally, an anode plasma may be formed [19].

Electrons are extracted from the plasma and accelerated in the remaining vacuum gap producing bremsstrahlung and characteristic radiation when they impinge on the anode. The electron current in the gap is essentially space-charge limited $[14,16]$. When the expanding cathode plasma cloud has reached the anode an arc develops. A fairly short circuit is formed and $\mathrm{x}$ ray emission is practically terminated. This effect is well known as plasma closure and typically occurs with cold cathodes. Hence, only the period before vacuum breakdown, in the arc formation period, is of interest for the production of $\mathrm{x}$-ray flashes. However, since most of the energy initially stored may be dissipated during the arc phase, the electrodes or other circuit elements such as spark gaps may be severely damaged. Arc formation should therefore be avoided by proper choice of tube and generator parameters $[2,10,18]$.

\section{Results}

\subsection{Parametric study}

The tube current, $x$-ray dose rate and total x-ray dose were measured as a function of the charging voltage, cathode size and anode-cathode separation. For not too large electrode separation $\left(d_{0} \leqslant 4 \mathrm{~cm}\right)$, the signals are very smooth; the shot-to-shot stability of the peak current is better than $\pm 1 \%$ and that of the PIN diode 


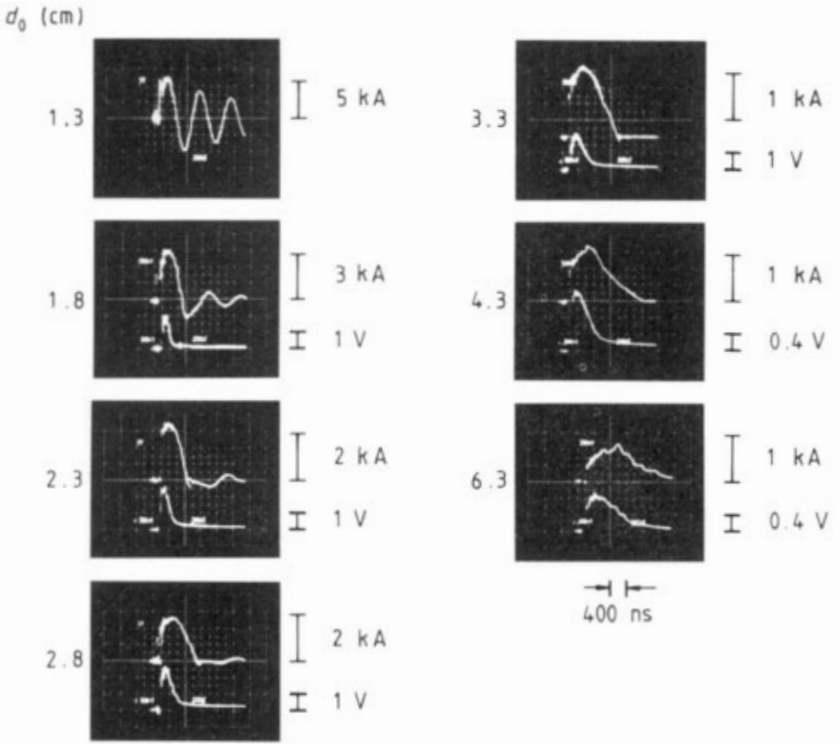

Figure 2. X-ray tube current (top) and $x$-ray dose rate (bottom) as traced by the PIN diode for various electrode separations $d_{0}$. Here the three-stage Marx generator was used with $U_{C}=30 \mathrm{kV}$ and $A=2 \times 40 \mathrm{~cm}^{2}$.

signal is better than $\pm 2.5 \%$. Figure 2 displays the diode current and the respective $x$-ray pulse shape for the small cathode and various anode-cathode distances $d_{0}$ with $U_{\mathrm{C}}=30 \mathrm{kV}$ (three-stage Marx generator). For $d_{0}=1.3 \mathrm{~cm}$, the current exhibits nearly short-circuit characteristics due to rapid arc formation across the gap. In this case, $x$-ray emission is low $(10 \mathrm{mR})$ and the PIN diode signal is very noisy. For distances up to $2.8 \mathrm{~cm}$, current oscillations are observed indicating the formation of a low-resistive arc after plasma closure. The amplitude of the current oscillations decreases for larger $d_{0}$ for two reasons. First, the resistance of the tube in the arc regime becomes larger with increasing $d_{0}$ causing a faster decay of the current oscillations [2]. Second, the portion of the total stored energy which is converted into kinetic energy of the electrons before the plasma has reached the anode will presumably increase with $d_{0}$. Consequently, the energy still available in the arc regime is expected to be smaller for larger $d_{0}$. For $d_{0} \geqslant 3.3 \mathrm{~cm}$, the electrical energy is deposited into the load within a single current pulse. For even larger $d_{0}$. the current trace becomes more and more irregular and the fluctuations from shot to shot become larger.

The $x$-ray pulse always peaks before the current maximum [2] which is a clear indication of the decreasing effective electrode separation. The current characteristics of the large cathode are similar to those of the small one. but the current is considerably higher and the duration of both current and x-ray pulse is shorter. The minimum electrode separation at which no current reversal occurs is practically equal for both cathode sizes.

In figure 3 , the peak tube current and the $x$-ray pulse duration (FWHM) are plotted as a function of the anode-cathode separation for the two different cathodes. With increasing distance, the peak current

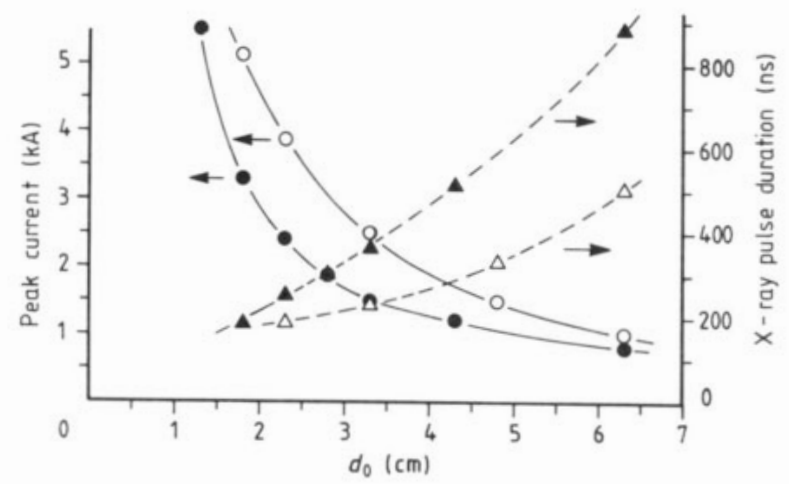

Figure 3. Peak tube current and $x$-ray pulse duration (FWHM) as a function of electrode separation $d_{0}$ (three-stage Marx generator, $U_{C}=30 \mathrm{kV}$ ) for two different cathode areas $A: A, A=2 \times 40 \mathrm{~cm}^{2}$;

$\mathrm{O}, \triangle, A=10 \times 40 \mathrm{~cm}^{2}$.

decreases in qualitative agreement with the spacecharge limited current between two plane parallel electrodes of area $A$ (Child-Langmuir law) [20]

$$
I={ }_{9}^{4} \varepsilon_{0}\left(\frac{2 e}{m_{\mathrm{c}}}\right)^{\mathrm{l} / 2} \frac{U_{\mathrm{v}}^{3 / 2}}{d^{2}} A=\alpha \frac{U_{\mathrm{V}}^{3 / 2}}{d^{2}} A
$$

where $I$ denotes the tube current, $d$ the electrode separation, $U_{\mathrm{V}}$ the voltage across the vacuum gap and $e$ $\left(m_{\mathrm{c}}\right)$ the charge (mass) of an electron. The spacecharge law in this simple form only applies if the work function of the cathode is negligible. Equation (1) does not consider the migration of the plasma front, which diminishes the effective electrode separation with time. The increase of the $\mathrm{x}$-ray pulse duration with $d_{0}$ as well as the delay between the maxima of $x$-ray intensity and current are indications for this movement.

Additionally, since the Marx generator is not a constant-voltage source, the time dependence of the acceleration voltage $U_{\mathrm{V}}$ has to be taken into account. As a consequence, the peak current is not proportional to the cathode area as may be concluded from equation (1) (figures 3 and 4). Hence, the ratio of the peak currents need not contradict the validity of the spacecharge law.

Visual inspection of the discharge shows a homogeneous distribution of several hundred sparks on the cathode, each representing an exploding whisker. The areal density of the exploding whiskers on the cathodes is so high that the single plasma clouds apparently overlap.

The cathode plasma not only expands directly towards the anode. but in a considerably larger solid angle (see also [14]). It is seen through the windows that a large number of sparks are formed at the sides of the carbon felt, i.e. the plasma also expands orthogonally to the anode-cathode gap. This will be recognised in the effective area of the tube current, which is larger than the physical cathode surface area (see $\$ 4.2$ ).

In figure 4 , the $x$-ray dose and the peak dose rate are plotted as a function of $d_{0}$, measured under the same experimental conditions as figure 3 . It is seen that both $\mathrm{x}$-ray dose rate and dose are considerably higher 


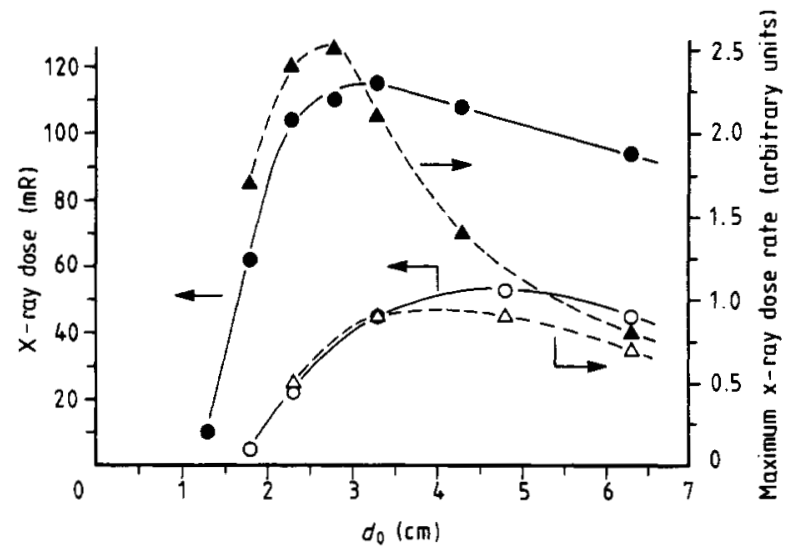

Figure 4. $X$-ray dose per pulse and peak $\mathrm{x}$-ray dose rate as a function of electrode separation $d_{0}$ (three-stage Marx generator, $U_{\mathrm{c}}=30 \mathrm{kV}$ ) for two different cathode areas $A$ (symbols as in figure 3 ).

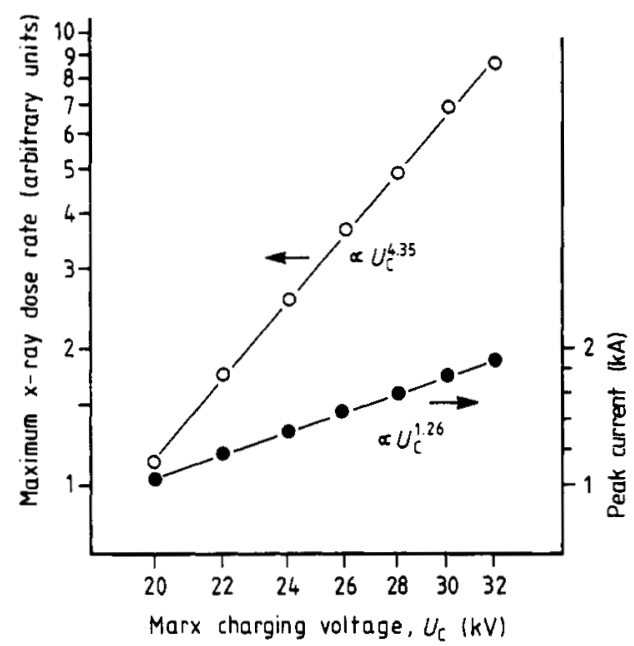

Figure 5. Dependence of the peak tube current (right-hand scale) and the peak $x$-ray dose rate (left-hand scale) on the Marx charging voltage $U_{C}$. Here a four-stage Marx generator and the small cathode $\left(A=2 \times 40 \mathrm{~cm}^{2}\right)$ were employed with $d_{0}=3.6 \mathrm{~cm}$.

for the small cathode. Although $j$ is explicitly independent of the cathode size (since the current is spacecharge limited), the higher total current of the large cathode leads to faster discharge of the Marx generator and consequently to a shorter $\mathrm{x}$-ray pulse. For the larger cathode, the whole $\mathrm{x}$-ray energy is distributed over a larger area. Hence, the dose produced by a certain area must be smaller.

For a given $d_{0}$, the peak tube current varies with the Marx charging voltage $U_{\mathrm{C}}$ in good agreement according to $U_{\mathrm{C}}^{x}$, with $1.0 \leqslant x \leqslant 1.3$, depending on the actual tube parameters. The experimental value for the exponent $x$ is not very different from the exponent 1.5 in the space-charge law (equation (1)) and is in agreement with the observation of other authors [13]. The dose $D$ as well as the peak x-ray dose rate (as observed by the pIN diode) follow a power law $\propto U_{\mathrm{C}}^{\mathrm{Y}}$, with $3.5 \leqslant y \leqslant 4.5$ (figure 5).

The strong dependence can qualitatively be understood by neglecting any temporal change of $U_{\mathrm{V}}$ or $d$. For not too high an acceleration voltage, the spectrally integrated x-ray intensity $S_{\text {cont }}$ (in units of $\mathrm{W} \mathrm{cm}^{-2}$ ) originating from bremsstrahlung radiation for a 'thick' anode is approximately proportional to [21]

$$
S_{\text {cont }} \propto j U_{\mathrm{V}}^{2}
$$

where $j$ is the current density. The intensity of characteristic radiation $S_{\text {char }}$ has been found to be proportional to $[10,22]$

$$
S_{\text {char }} \propto j\left(U_{\mathrm{V}}-U_{\mathrm{I}}\right)^{\beta} \quad 1.5 \leqslant \beta \leqslant 2.0 .
$$

$U_{\mathrm{I}}$ denotes the ionisation energy of a particular inner electron. $\mathbf{L}$ radiation is immediately reabsorbed in the Ta anode. Using the constants for equations $(2 a, b)$ given in $[21,22]$ and assuming a constant voltage of $120 \mathrm{kV}$ it is seen that the spectrally integrated intensity of continuous radiation is nearly two orders of magnitude larger than that of the $\mathrm{K}$ line. Since the temporally varying tube voltage generally exceeds the $\mathrm{K}$ binding energy only for a short time this ratio will be even higher. Hence, it is reasonable to neglect characteristic radiation, as we will do in the following.

It follows from equations (1) and (2) that the x-ray intensity is only a function of the current density $j$. Since $j \propto U_{\mathrm{V}}^{3 / 2}, S \propto U_{\mathrm{V}}^{7 / 2}$. Since a slightly shorter x-ray pulse is expected at higher driving voltage in cold cathode tubes, an $S \propto U_{\mathrm{C}}^{3}$ law was derived in [4]. The dependence in the present work is in general significantly stronger. The reason for this is not fully understood, but we believe that it is partly due to the spectral composition of the radiation which is not exactly known. The radiation is, for example, filtered by the $3 \mathrm{~mm}$ thick aluminium window of the tube. It can be easily shown that the voltage dependence of the $\mathrm{x}$-ray intensity becomes stronger if the radiation is filtered.

Additionally, the difference between $\mathrm{x}$-ray intensity and $\mathrm{x}$-ray dose rate should be kept in mind. Equation (2a) gives a value for the spectrally integrated $\mathrm{x}$-ray intensity (in $\mathrm{W} \mathrm{cm}^{-2}$ ) of the emitted radiation. However, the pen dosemeter and the PIN diode measure the energy (power) of the radiation absorbed in a thin layer of air (silicon, respectively). The factor between intensity and dose rate is strongly wavelength dependent through the photoelectric cross section [23]. The results of the dose measurements and the diode signal are in full agreement with each other and, therefore, we consider the strong voltage dependence to be an experimental fact.

A more detailed analysis would require further assumptions about or accurate measurements of the $x-$ ray spectrum, which have not yet been performed. However, the $\mathrm{x}$-ray spectrum could be estimated from the attenuation coefficient of the radiation in pure copper plates of various thickness. With a four-stage Marx generator and $U_{\mathrm{C}}=35 \mathrm{kV}$, the intensity spectrum extends roughly from 20 to $90 \mathrm{keV}$ and peaks at about $30 \mathrm{keV}$ (figure 6).

The voltage across the tube can be considerably increased by a peaking capacitor $C_{\mathrm{p}}$ which is small compared with the erected capacitance of the Marx 


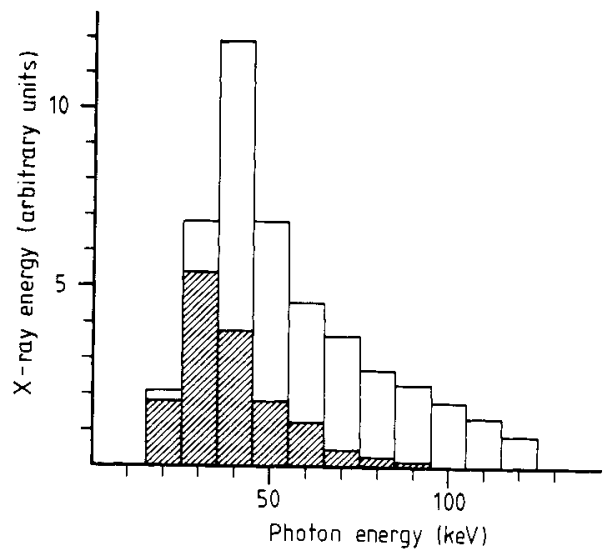

Figure 6. Schematic spectral distribution of the x-ray energy without (hatched) and with peaking capacitor (four-stage Marx, $U_{\mathrm{C}}=35 \mathrm{kV}, d_{0}=2.1 \mathrm{~cm}$ ).
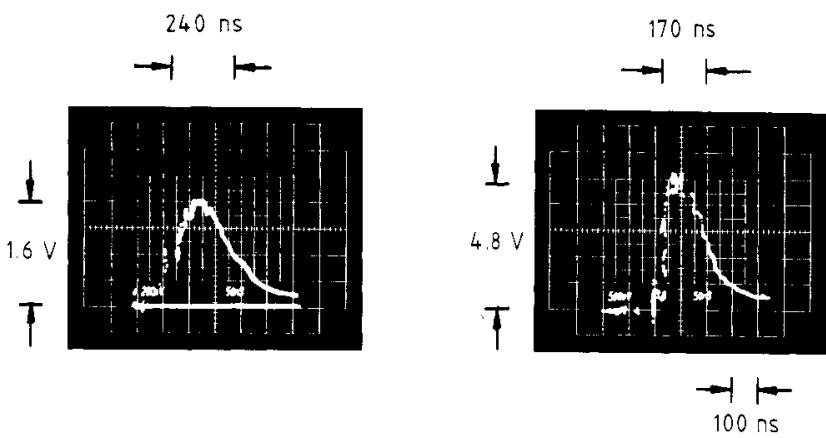

Figure 7. Comparison of the $x$-ray dose rate (as traced by the PIN diode) without (left) and with the use of an additional peaking capacitor, $C_{\mathrm{p}}=4 \mathrm{nF}$ (three-stage Marx generator, $\left.U_{C}=30 \mathrm{kV}, d_{0}=2.3 \mathrm{~cm}\right)$.

generator [6]. Furthermore, the inductance of the discharge loop is decreased to about $500 \mathrm{nH}$. Best performance was obtained with $C_{\mathrm{p}}=4 \mathrm{nF}$. Depending on the gap size, an increase of the $x$-ray dose rate up to a factor of three and a pulse length reduction of up to $60 \%$ could be achieved (figure 7). Additionally, the pulse dose was typically increased by about $100 \%$ with $C_{\mathrm{p}}$. The x-ray spectrum peaks at $40 \mathrm{keV}$ and extends to $120 \mathrm{keV}$ with $C_{\mathrm{p}}$ (figure 6). The spectrally integrated $\mathrm{x}$-ray energy is about three times as high as without $C_{\mathrm{p}}$.

The x-ray flash tube has been used successfully for the preionisation of rare-gas halide lasers of different volumes $[5,24]$. In order to avoid unnecessary stress of the tube electrodes by arc formation we chose $d_{0}=$ $3.3 \mathrm{~cm}$ and the small cathode for continuous use. As can be seen from figure 4 , this specification corresponds to a maximum value for the $\mathrm{x}$-ray dose. The tube has been reliably operated for several tens of thousands of discharges.

\subsection{X-ray tube model}

It is well known that the effective impedance $Z$ of a cold cathode $x$-ray tube decreases rapidly after discharge initiation [2]. Empirically, this decay can be well described by a power law, $Z \propto t^{-\alpha}$, where $\alpha \approx$ $2-3$ [10]. The region of smallest impedance already

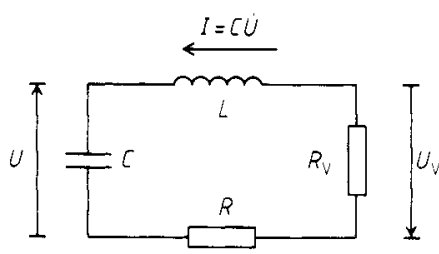

Figure 8. Equivalent circuit used for tube simulation.

belongs to the arc phase. However, this empirical description is not fully satisfactory, particularly in the pre-breakdown phase. We want to show in the present paper that the assumption of a cathode plasma moving with constant velocity and having an effective area linearly growing with time and a space-charge limited current in the remaining vacuum gap between the anode and the plasma is in full agreement with the experimental observations.

The equivalent circuit employed for modelling the $x$-ray flash system is shown in figure 8 . No peaking caprcitor was used in these experiments. For reasons of simplicity, the Marx generator is represented by a capacitor $C$ charged to $U_{0}$ at $t=0 . L$ and $R$ are the time-independent inductance and ohmic resistance of the whole circuit. The cathode plasma expands with a constant velocity $v . R_{\mathrm{V}}\left(=U_{\mathrm{V}} / I\right)$ is the non-linear resistance of the vacuum gap (equation (1)).

The plasma is formed at the beginning of the discharge by local heating caused by the field-emission current. Afterwards, it expands and therefore it is cooled and diluted. However, it is heated by ohmic losses (otherwise the electrons would be accelerated to several $10 \mathrm{keV}$ within the plasma) and the electron density increases with time [17]. Because of charge neutrality, the current through the plasma is not spacecharge limited. Assuming an ion density of $10^{17} \mathrm{~cm}^{-3}$, a temperature of $10000 \mathrm{~K}$ and an effective ion charge of 2 (as was measured in plasmas originating from graphite cathodes [17]), we estimate the specific resistance by a model for a completely ionised plasma (Lorentz gas) [25] to be less than $0.1 \Omega \mathrm{cm}$. With a typical plasma area of $300 \mathrm{~cm}^{2}$ and a length of $3 \mathrm{~cm}$, the resistance of the plasma becomes $\approx 1 \mathrm{~m} \Omega$, which is completely negligible compared with the impedance of the vacuum gap (equation (1)) and the impedance of the remaining circuit $(\sqrt{L / C} \approx 20 \Omega)$.

Because of the symmetry of the cathode, it is reasonable to assume a simple linear growth of the effective cathode plasma surface with time, i.e. $A=$ $A_{0}+\beta t[7,14]$. Anode plasma formation is not considered. The vacuum gap reaches from the front of the cathode plasma to the anode and represents a nonlinear real impedance $R_{\mathrm{V}}$. Since the current in the gap is space-charge limited, the $I-U_{\mathrm{V}}$ characteristic is given by the Child-Langmuir law (equation (1)). The plasma is assumed to have a negligible work function.

The equation of the circuit finally reads

$$
U+L C \frac{\mathrm{d}^{2} U}{\mathrm{~d} t^{2}}+R C \frac{\mathrm{d} U}{\mathrm{~d} t}+\left(\frac{C d^{2}}{\alpha A} \frac{\mathrm{d} U}{\mathrm{~d} t}\right)^{2 / 3}=0
$$

with $A=A_{0}+\beta t$ and $d=d_{0}-v t$. $U$ is the voltage on 
Table 1. The parameter set that describes the discharge kinetics of the present $x$-ray flash tube (small cathode). $L$ is the circuit inductance, $v$ the plasma velocity, $A=A_{0}+\beta t$ the effective area of the cathode plasma and $R$ the ohmic resistance of the whole circuit.

$$
\begin{aligned}
& L=3.02 \pm 0.05 \mu \mathrm{H} \\
& V=3.41 \pm 0.06 \mathrm{~cm}_{\mu \mathrm{s}^{-1}} \\
& A_{0}=285 \pm 25 \mathrm{~cm}^{2} \\
& \beta=280 \pm 135 \mathrm{~cm}^{2} \mu \mathrm{s}^{-1} \\
& R=0.95 \pm 0.04 \Omega
\end{aligned}
$$

the erected Marx capacitance. The initial conditions are $U(t=0)=U_{0}$ and $I(t=0)=C \mathrm{~d} U / \mathrm{d} t=0 . U_{0}, C$, $L, R$ and $d_{0}$ can be directly measured or derived from other measurements with an uncertainty of about $\pm 2 \%$ ( $L$ and $R$ can be derived from the period and the damping constant of the short circuit current oscillations putting the cathode in direct contact with the anode). The remaining parameter set, consisting of $A_{0}$, $\beta$ and $v$, is determined so that the values are constant for the different experiments where $C$ and $d_{0}$ are varied.

Since by changing each of the three free parameters the current waveform is modified in a characteristic way, the fitting procedure is quite stable and the derived values for $A_{0}, \beta$ and $v$ can be considered as reliable within the intervals shown in table 1 . Since $R \ll \sqrt{L / C}$, the choice of $R$ does not have a very critical influence on the calculated current curves.

Equation (3) is solved on a personal computer using the numeric data from the 7250 digital oscilloscope. In these measurements, the $2 \mathrm{~cm}$ wide cathode was employed. The parameter values were obtained by a least-squares fit to the experimental current data between $t=0$ and $t=t_{\mathrm{C}}$, the time of plasma closure or $t_{R}$, the time of current reversal, whichever occurs first. The aim of this work is to simulate the tube current and the $\mathrm{x}$-ray intensity before these events. A description of the circuit after plasma closure (in the arc phase) requires a completely different model. Moreover, after current reversal (which may occur before plasma closure) electron emission from the anode has to be considered. This will probably occur through field emission, but the work function of the anode cannot be neglected. Therefore, equation (3) is not able to predict correctly the tube behaviour for either $t>t_{\mathrm{C}}$ or for $t>t_{\mathrm{R}}$.

In figures 9 and 10 the calculated temporal behaviour of tube current and $\mathrm{x}$-ray intensity is compared with the experimental data for two different electrode separations $d_{0}$. The $\mathrm{x}$-ray intensity $S$, plotted in arbitrary but equal units, is assumed to be given according to equation $(2 a)$.

Figure 9 shows the situation for a four-stage Marx generator, $U_{\mathrm{C}}=30 \mathrm{kV}$ and $d_{0}=2.1 \mathrm{~cm}$. The arrow marks the moment of plasma closure assuming a plasma velocity of $3.4 \mathrm{~cm} \mu \mathrm{s}^{-1}$ by which the experimental data are fitted best. It is seen that here $t_{\mathrm{R}}$ and

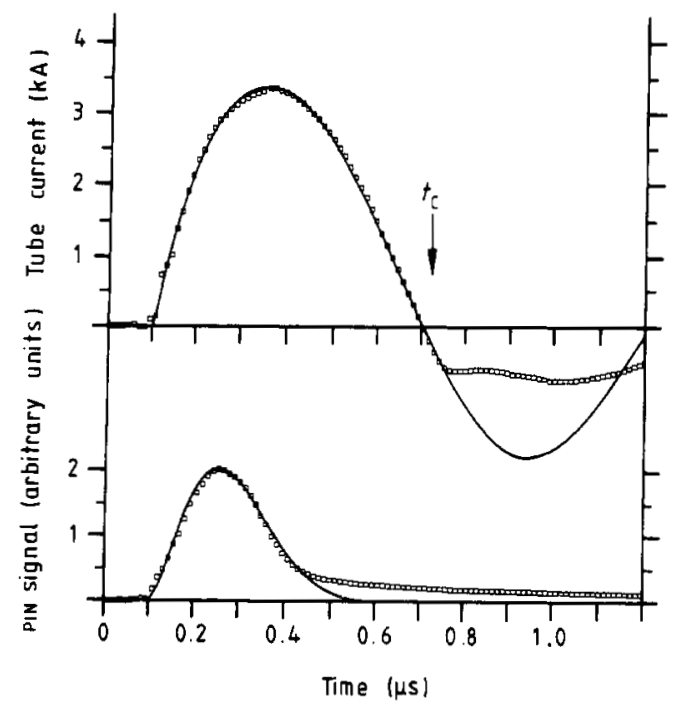

Figure 9. Experimental traces of the tube current (top) and the $x$-ray dose rate (bottom) fitted by a solution of equation (3). A four-stage Marx generator and the small cathode were employed, $d_{0}=2.1 \mathrm{~cm}$.

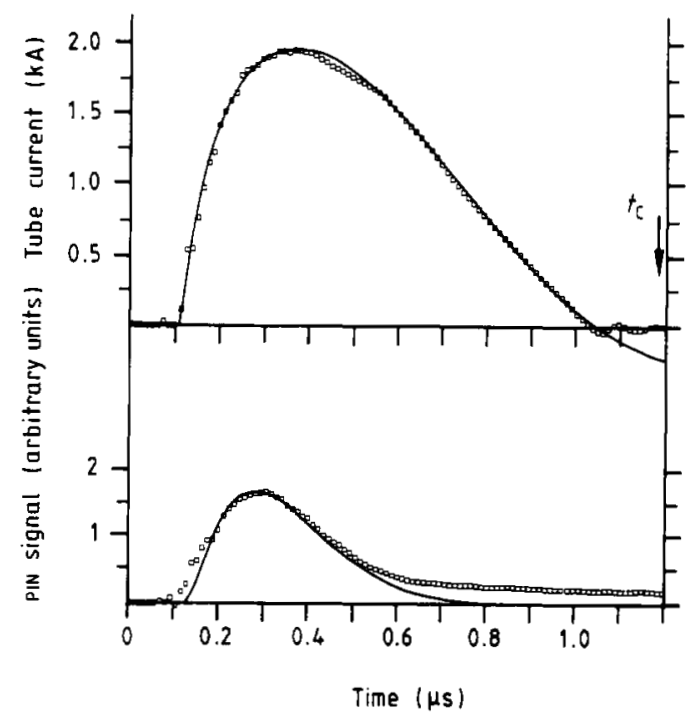

Figure 10. Same as figure 9 , but for $d_{0}=3.6 \mathrm{~cm}$.

$t_{\mathrm{C}}$ approximately coincide. Since at $t=t_{\mathrm{C}}$ there is still some energy stored in the capacitors, an arc is formed which is indicated by current oscillations.

For $d_{0}=3.6 \mathrm{~cm}$ (figure 10 ) plasma closure occurs more than $150 \mathrm{~ns}$ after the current has reached the zero

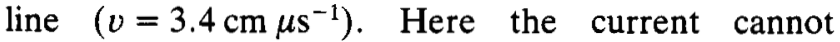
reverse, since electron emission by the anode is not possible. Hence the tube current is abruptly terminated. This also shows that practically no anode plasma is formed before plasma closure. In this case also the voltage at the high-voltage feedthrough was measured and compared with the calculation. As well as the current trace, the voltage waveform is abruptly terminated. As an indication of the plasma movement (decrease in $d$ ) it peaks earlier than the current and even the $x$-ray pulse [2]

The time response of the voltage- divider is apparently not fast enough to resolve the initiation of the discharge. Immediately after the erection of the Marx 
generator the tube voltage should be rather high (since no current can flow before the cathode plasma has been formed). The duration of plasma formation has been observed to be $10-100$ ns with graphite cathodes [14]. In the present tube, the time of formation appears to be definitely shorter than the $50 \mathrm{~ns}$ time resolution of the divider, which we attribute to facilitated plasma formation of the carbon felt cathode.

The $\mathrm{x}$-ray pulse shape is quite satisfactorily predicted by the simulation in spite of the discrepancy between intensity and dose rate (see §4.1). Apparently, this difference does not very strongly affect the pulse shape in practical terms. The experimental $x$-ray trace has a tail of about $1 \mu \mathrm{s}$ which is not predicted. This is an artefact (presumably caused by slow diffusion of charge carriers in the diode), since there is still an $\mathrm{x}$-ray signal although the tube current has ceased (figure 10). It has been checked that the rise time of the PIN diode is of the order of $10 \mathrm{~ns}$.

The voltage dependence of the peak current is in excellent agreement with the experimental data when a constant parameter set is used $\left(I_{\max } \propto U_{\mathrm{C}}^{y}, 1.2 \leqslant y\right.$ $\leqslant 1.3$ ), and also the dependence of $I_{\max }$, the dose and maximium dose rate on $d_{0}$ (figures 3 and 4 ) are in good agreement with the experiment. However, the calculated dependences of the dose and the peak dose rate on the charging voltage $U_{\mathrm{C}}$ are too weak compared with experiment. Both are predicted by the model to vary approximately with the third power of $U_{C}$. The dose increases slightly slower than the peak dose rate due to a somewhat shorter pulse length. As has been mentioned in $\S 4.1$, the source of this discrepancy is not fully understood.

The current traces for various electrode separations $d_{0}$ and for two different Marx generator designs ( $n=$ $3,4)$ have been described by equation (3). A consistent parameter set could be derived by least-squares fits (table 1)

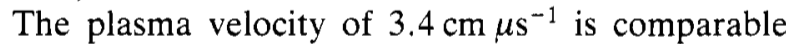
with values reported by other authors [14]. The expansion rate of the plasma area corresponds to a reasonable emission angle of $60 \pm 10^{\circ}$, measured from the forward direction. There may be some doubt about the large initial area $A_{0}$ which is more than three times larger than the physical area of carbon felt $\left(80 \mathrm{~cm}^{2}\right)$. However, this large emission area is real as has been checked by measuring the dose along a line parallel to the short dimension of the cathode below the output window. The width (FWHM) of the active anode area is usually of the order of $6-8 \mathrm{~cm}$. Possibly plasma formation at the side surfaces of the carbon felt cathode precedes the formation on the surface facing towards the anode and so increases the initial effective emission area.

\section{Conclusion}

A simple and reliable $\mathrm{x}$-ray flash source designed for preionising gas discharge lasers is described. Output dose rate and dose are strongly dependent on the anode-cathode separation, the cathode area and the operating voltage. The tube can be adjusted by simple geometrical changes so that no arc is formed which might damage the electrodes.

The $\mathrm{x}$-ray tube provides a smooth and stable $\mathrm{x}$-ray output $( \pm 5 \%)$. It can be described in good agreement with experiment by assuming a cathode plasma with an effective area linearly growing in time and a constant velocity of about $3.4 \mathrm{~cm} \mu \mathrm{s}^{-1}$. The current in the remaining vacuum gap is space-charge limited.

The plasma movement is clearly indicated by a time delay between the maxima of tube voltage, x-ray pulse and current. However, some open questions remain, like the strong voltage dependence of the $\mathrm{x}$-ray output.

The $\mathrm{x}$-ray flash tube has been operated during several tens of thousands of shots to preionise the $30 \mathrm{~cm}^{3}$ discharge volume of a rare-gas halide laser. The influence of gas composition, preionisation geometry, $\mathrm{x}$-ray dose, pressure and time delay between the $x$-ray pulse and the laser discharge on the output energy has been extensively studied [5]. In a modified version it is currently in operation with another $\mathrm{XeCl}$ excimer laser having a discharge volume of about $5 \times 6 \times 40 \mathrm{~cm}^{3}$, from which $5 \mathrm{~J}$ in a $45 \mathrm{~ns}$ pulse could be extracted [24].

\section{Acknowledgments}

The author would like to thank Professor F P Schäfer for continuous support and stimulating interest. The contributions of Dr K A Stankov, Dr H Voges, $\mathrm{H}$ Mizoguchi and B Ouyang are gratefully acknowledged.

\section{References}

[1] Germer R 1979 J. Phys. E: Sci. Instrum. 12 337-50

[2] Jamet F and Thomer G 1976 Flash Radiography (New York: Elsevier) pp 15-85

[3] Sumida S, Obara M and Fujioka T 1978 Appl. Phys. Lett. 33 913-5

[4] Lin S C, Zheng C E, Lo D and Matsumoto J 1986 Appl. Phys. B 40 15-23

[5] Steyer M and Voges H 1987 Appl. Phys. B 42 155-60

[6] Jayaram K and Alcock A J $1985 \mathrm{~J}$. Appl. Phys. 58 1719-26

[7] Bonnie R J M, Gerritsen J W and Zuidema R J 1989 J. Appl. Phys. 65 4521-8

[8] Thomer G 1967 Röntgenblitztechnik in Kurzzeitphysik ed. K Vollrath and G Thomer (Vienna: Springer) pp 328-66

[9] Eden J G and Epp D 1980 Rev. Sci. Instrum. 51 781-5

[10] Krehl P 1986 Rev. Sci. Instrum. 57 1581-9

[11] Goldman M and Goldman A $1963 J$. Physique 24 303-6

[12] Händel S K and Sundström I 1965 Z. Phys. 188 506-18

[13] Johnson Q, Mitchell A C and Smith I D 1980 Rev. Sci. Instrum. 51 741-9

[14] Parker R K, Anderson R E and Duncan C V 1973 J. Appl. Phys. 45 2463-79

[15] Chatterton P A 1978 Vacuum breakdown in Electrical Breakdown of Gases ed. J M Meek and J D Craggs (Chichester: Wiley) pp 129-208 
[16] Miller R B 1982 An Introduction to the Physics of Intense Charged Particle Beams (New York: Plenum) pp 31-76

[17] Hinshelwood D D 1983 IEEE Trans. Plasma Sci. PS-11 188-96

[18] Lafferty J M 1987 Vacuum arcs in Encyclopedia of Physical Science and Technology vol 14 (New York: Academic) pp 249-61

[19] Adolfs F, Müller F and Schulte E 1978 J. Phys. E: Sci. Instrum. 11 537-40

[20] Child C D 1911 Phys. Rev. 32 492-511

Langmuir I 1914 Phys. Z. 15 348-53
[21] Agarwal B K 1979 X-ray Spectroscopy (Berlin: Springer)

[22] Green M and Cosslett V E 1968 Br. J. Appl. Phys. $1425-36$

[23] Robinson J W (ed.) 1974 Handbook of Spectroscopy vol I (Cleveland: CRC)

[24] Steyer M, Stankov K A, Mizoguchi H, Ouyang B and Schäfer F P 1989 Appl. Phys. B 49 331-7

[25] Alexandrov A F, Bogdankevich L S and Rukhadze A A 1984 Principles of Plasma Electrodynamics (Berlin: Springer) pp 66-7 